\title{
Improved parametrization of the transparency parameter in Kox and Shen models of total reaction cross sections
}

\author{
L. Sihver, ${ }^{1, *}$ M. Lantz, ${ }^{2,3}$ and A. Kohama ${ }^{3}$ \\ ${ }^{1}$ Nuclear Engineering, Applied Physics, Chalmers University of Technology, Gothenburg, Sweden \\ ${ }^{2}$ Applied Nuclear Physics, Department of Physics and Astronomy, Uppsala University, Uppsala, Sweden \\ ${ }^{3}$ RIKEN Nishina Center, RIKEN, Wako, Saitama 351-0198, Japan
}

(Received 17 March 2014; revised manuscript received 19 May 2014; published 30 June 2014)

\begin{abstract}
The total reaction cross section is an essential quantity in particle and heavy-ion transport codes when determining the mean free path of a transported particle. Many transport codes determine the distance a particle is transported before it collides with the target or is stopped in the target material, with the Monte Carlo (MC) method using semiempirical parametrization models for the total reaction cross sections. In order to improve the well-known Kox and Shen models of total reaction cross sections and allow the models to be used at energies below $30 \mathrm{MeV} /$ nucleon, we propose a modified parametrization of the transparency parameter. We also report that the Kox and Shen models have a projectile-target asymmetry and should be used so that the lighter nucleus is always treated as the projectile.
\end{abstract}

DOI: 10.1103/PhysRevC.89.067602

PACS number(s): 24.10.Lx, 25.40.-h, 25.70.-z, 25.75.-q

Introduction. Accurate knowledge of the nucleon-nucleus and nucleus-nucleus total reaction cross sections is of importance for many different fields including fundamental nuclear physics, simulations of radiation damage to equipment used on space vehicles, and transport of the heavy ions in the galactic cosmic rays (GCR) through the interstellar medium, as well as to estimate the biological effects of space radiation. During the past decades, new applications have also arisen within transmutation, reactor science, and medicine, especially in radiotherapy with protons and ions. Several medical accelerator facilities are operating or are planned for construction. In order to be able to calculate complex geometries, including production and transport of protons, neutrons, $\alpha$ particles, and heavy ions in different materials, including human tissues and organs, three-dimensional Monte Carlo (MC)-based transport codes must be used. The total reaction cross section and the decay lifetime of the particle are essential quantities when determining the mean free path of a transported particle. Many particle and heavy-ion transport codes choose the next collision point using the MC method and the calculated total reaction cross section. Due to calculation speed, simplicity, and accuracy, semiempirical parametrization models for the total reaction cross section are most often used in these codes.

During the 1980s, Kox and coworkers performed a series of experiments where the total reaction cross sections were measured over a wide energy range for ${ }^{12} \mathrm{C}$ and ${ }^{20} \mathrm{Ne}$ on different target materials [1-4]. Besides generally supporting the development of microscopic models for the prediction of total reaction cross sections, they also developed their own general parametrization, commonly referred to as the Kox model, that was valid for many different projectiles at energies above $30 \mathrm{MeV} /$ nucleon [4]. The Kox model follows the semiempirical Bradt-Peters form [5]. A few years later the model was improved by Shen et al. [6], by adjusting the parameters and extending its validity to energies below $30 \mathrm{MeV} /$ nucleon. However, the energy-dependent transparency parameter, $C(E)$, is not given in numerical or functional form in the article but is only shown in a figure. Furthermore, the Shen model gives different results for some of the systems where the projectile and target nuclei have different masses, if run in the inverse kinematics. Nevertheless, the models by Kox and Shen are still available as options in several Monte Carlo particle and transport codes.

In this paper a modified and improved parametrization of the transparency parameter in the Kox and Shen models is therefore presented. The main improvement with our parametrization compared to the original ones is that it results in a smoother energy dependence of the total reaction cross sections and extends the models to be used for projectile energies below $30 \mathrm{MeV} /$ nucleon. We also report that the Kox and Shen models have a projectile-target asymmetry and we therefore give the recommendation that the models should be used so that the lighter nucleus is always treated as the projectile, no matter what the actual case might be.

It should be pointed out that there are several parametrizations of later date that in general give a more accurate prediction of the total reaction cross sections, e.g., the model developed by Tripathi et al. [7-9] and the empirically modified version [10] of the Tripathi model used in the three-dimensional particle and heavy-ion transport code FLUKA $[11,12]$. Other promising models used in different particle and heavy ion transport codes are the Sihver model $[13,14]$ and the models developed by Takechi et al. [15] and Iida et al. [16]. However, it should be pointed out that there is no current model which agrees well with the experimental data for all possible nucleon-nucleus and nucleus-nucleus collisions in the energy range $10-10^{4} \mathrm{MeV} /$ nucleon.

The transparency parameter. The Kox and Shen models are based on the strong absorption model and have the same general form:

$$
\sigma_{R}=\pi R^{2}\left[1-\frac{B}{E_{C M}}\right]
$$


where $R$ is the interaction radius and $E_{C M}$ is the center-of-mass energy of the collision. The term $B$, which describes the effects of the Coulomb barrier, has a slightly different form in the two models:

$$
\begin{aligned}
B_{\text {Kox }} & =\frac{Z_{\mathrm{p}} Z_{\mathrm{t}} e^{2}}{1.3\left(A_{\mathrm{p}}^{1 / 3}+A_{\mathrm{t}}^{1 / 3}\right)}, \\
B_{\text {Shen }} & =\frac{1.44 Z_{\mathrm{p}} Z_{\mathrm{t}} e^{2}}{R_{\mathrm{p}}+R_{\mathrm{t}}+3.2}-\frac{R_{\mathrm{p}} R_{\mathrm{t}}}{R_{\mathrm{p}}+R_{\mathrm{t}}}, \\
R_{i} & =1.12 A_{i}^{1 / 3}-0.94 A_{i}^{-1 / 3}(i=\mathrm{p}, \mathrm{t}),
\end{aligned}
$$

where $A_{\mathrm{p}}, A_{\mathrm{t}}, Z_{\mathrm{p}}, Z_{\mathrm{t}}, R_{\mathrm{p}}$, and $R_{\mathrm{t}}$ are the mass numbers, charges, and radii of the projectile $(\mathrm{p})$ and target $(\mathrm{t})$, respectively. For simplicity, the values of all fixed parameters are here included in the equations. The complete expressions can be found in the original articles [4,6]. The forms for the interaction radii, $R$, are the same, except that the Shen model has one extra term,

$$
\begin{aligned}
R_{\text {Kox }}= & 1.1\left(A_{\mathrm{p}}^{1 / 3}+A_{\mathrm{t}}^{1 / 3}+1.85 \frac{A_{\mathrm{p}}^{1 / 3} A_{\mathrm{t}}^{1 / 3}}{A_{\mathrm{p}}^{1 / 3}+A_{\mathrm{t}}^{1 / 3}}-C(E)\right) \\
& +\frac{5\left(A_{\mathrm{t}}-2 Z_{\mathrm{t}}\right) Z_{\mathrm{p}}}{A_{\mathrm{p}} A_{\mathrm{t}}} \\
R_{\text {Shen }}= & R_{\text {Kox }}+0.176 E_{C M}^{-1 / 3} \frac{A_{\mathrm{p}}^{1 / 3} A_{\mathrm{t}}^{1 / 3}}{A_{\mathrm{p}}^{1 / 3}+A_{\mathrm{t}}^{1 / 3}}
\end{aligned}
$$

It should be noted that the last term in Eq. (4), the socalled neutron excess term, is only used at energies below $200 \mathrm{MeV} /$ nucleon in the Kox model, with the motivation that at higher energies the $n-p$ total cross section becomes equal to the $p-n$ and $p$ - $p$ total cross sections. In the Shen model the neutron excess term is used also at energies above $200 \mathrm{MeV} /$ nucleon. $C(E)$ is the energy-dependent transparency term and $E$ is the energy per nucleon in the laboratory system. In the Shen model the transparency parameter is denoted $C^{\prime}(E)$, in the original paper [6], because it is extended to energies below $30 \mathrm{MeV} /$ nucleon. It is, however, called $C(E)$ throughout this paper.

As seen from Eqs. (1)-(4), it is, besides the Coulomb correction term, $B$, for low energies, the transparency parameter that gives the energy dependence for the total reaction cross section in the Kox model. In the Shen model there is also a slight contribution to the energy dependence from Eq. (5). For the Kox model a number of values are given for the transparency parameter, as function of different energies in the energy range 30-2100 MeV/nucleon. Figure 1 shows the values for $C(E)$ given by Kox et al. as black dots. The simplest way to determine the transparency parameter at other energies is by straight line interpolation between the given values. In Fig. 1 this is shown as a dashed black line.

Townsend and Wilson [17] have proposed an energydependent parametrization of the transparency parameter in the following way:

$$
C(E)=1.91-16.0 e^{-0.7274 E^{0.3493}} \cos \left(0.0849 E^{0.5904}\right),
$$

where $E$ is the projectile kinetic energy given in $\mathrm{MeV} /$ nucleon in the laboratory system. This parametrization is based on the

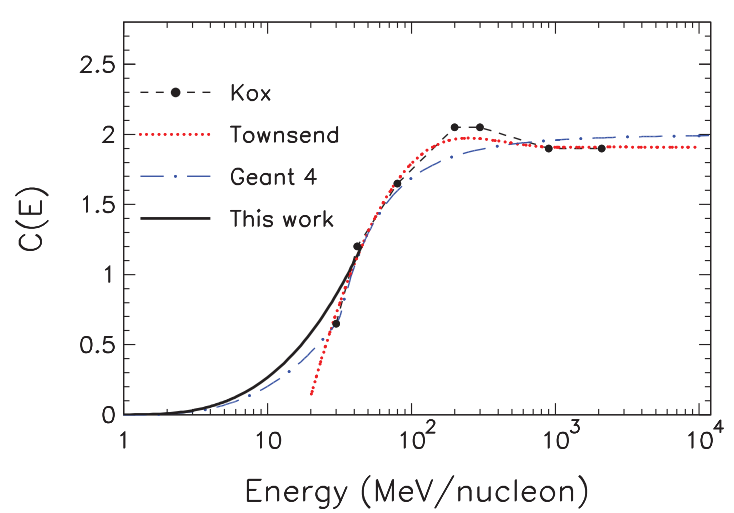

FIG. 1. (Color online) The $C(E)$ parameter as given by Kox (black dots), and a simple straight line interpolation between the dots (dashed black line). The dotted (red) line is the parametrization by Townsend and Wilson according to Eq. (6), and the dash-dotted (blue) line is the Shen model as it is used in GEANT4, using Eqs. (7) and (8). The proposed modified parametrization, using a combination of Eqs. (6) and (9) (see the text for details), is given as a solid black line. The line for the Townsend and Wilson model has been extrapolated somewhat below the lower limit of its given validity in order to show its behavior for lower energies.

$C(E)$ values by Kox [4] and gives good agreement with the Kox data above $30 \mathrm{MeV} /$ nucleon, while it is not defined below this energy range. At $30 \mathrm{MeV} /$ nucleon it agrees within $10 \%$, and at higher energies within 5\%. In Fig. 1 the parametrization by Townsend and Wilson is shown as a dotted (red) line.

The simple form of the parametrization by Townsend and Wilson, and the resulting smooth shape, makes it attractive for use in simulation codes where parametrized total reaction cross sections are required. As mentioned above, it has the drawback of not being defined at energies below $30 \mathrm{MeV} /$ nucleon. In Fig. 1 the curve is drawn to somewhat lower energies in order to show the trend if used below $30 \mathrm{MeV} /$ nucleon.

Besides the extra term in Eq. (5), and a few modified parameters which increased the validity of the model to more projectile-target systems, the main improvement of the Shen model was the extension to energies below $30 \mathrm{MeV} /$ nucleon; see Fig. 2 in Ref. [6]. But the extension of $C(E)$ is not given in any numerical or functional form. Attempts to extract values from the figure should be discouraged for two reasons:

(i) The figure seems to be drawn by hand, and close inspection shows that the curve above $30 \mathrm{MeV} /$ nucleon does not agree fully with the values of $C(E)$ given by Kox et al. [4]. Therefore there is no reason to believe that the suggested curve below $30 \mathrm{MeV} /$ nucleon can be extracted in a way that agrees with the intention of the authors.

(ii) The energy scale is inconsistent. Most likely it is the marker for the energy $10^{3} \mathrm{MeV} /$ nucleon that has been accidentally placed at the position for $2 \times 10^{3} \mathrm{MeV} /$ nucleon.

In the Monte Carlo simulation toolkit GEANT4 [18-20] the Kox and Shen models can be selected among other models. 


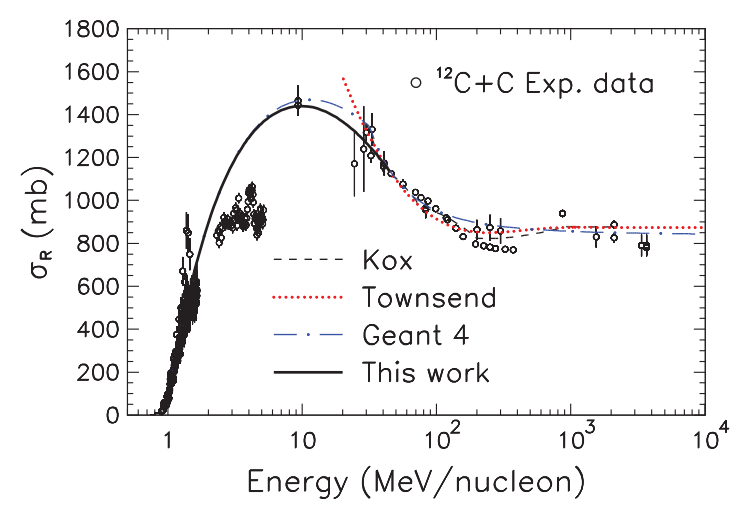

FIG. 2. (Color online) Comparison of the energy dependence of total reaction cross sections for ${ }^{12} \mathrm{C}$ on $\mathrm{C}$ calculated with the Kox and Shen models. The dashed black line is the Kox model, and the dotted (red) line is the result of the Kox model by using the Townsend and Wilson parametrization of $C(E)$. The dash-dotted (blue) line is the Shen model with the approach from GEANT4, and the solid black line is the modified parametrization presented in this work. Experimental data, shown as open circles, are taken from Ref. [21].

The authors of the code have defined $C(E)$ as

$$
\begin{aligned}
& C(E)=\left(-\frac{10}{1.5^{5}}+2.0\right)\left(\frac{x}{1.5}\right)^{3} \text { for } x<1.5, \\
& C(E)=-\frac{10}{x^{5}}+2.0 \text { for } x \geqslant 1.5
\end{aligned}
$$

where $x=\log _{10}(E)$. The shape of $C(E)$ is shown in Fig. 1 as a dash-dotted (blue) line. As seen it does not reproduce the peak values at about $200 \mathrm{MeV} /$ nucleon, and it gives a rather sharp corner where the two parts merge at about $31.6 \mathrm{MeV} /$ nucleon. We therefore propose a new parametrization where we use a modified version of Eq. (7) from the GEANT4 model, that gives a smooth overlap with the Townsend and Wilson model, Eq. (6), at $E=45 \mathrm{MeV} /$ nucleon, i.e.,

$$
C(E)=\left(-\frac{10}{1.5^{5}}+2.0\right)\left(\frac{x}{1.38}\right)^{3}+0.0006 E,
$$

where $E$ is the projectile energy per nucleon in the laboratory system. In Fig. 1 this version is shown as a solid black line for energies below $45 \mathrm{MeV}$ /nucleon.

Results. The different models discussed previously have been checked for a large number of cases together with available experimental data. Figure 2 shows the total reaction cross section for ${ }^{12} \mathrm{C}$ ions on $\mathrm{C}$ targets, together with experimental data from Ref. [21]. Figures 3 and 4 show the same situation for the systems ${ }^{12} \mathrm{C}+{ }^{27} \mathrm{Al}$ and ${ }^{12} \mathrm{C}+\mathrm{Fe}$, respectively. As seen our approach gives a smooth overlap with the Townsend and Wilson model and thus a smooth energy dependence over the full energy range.

It should be noted that our approach does not change the predictions at very low energies, and no modification has been attempted in order to give a better agreement with experimental data. This would require a modification of the Coulomb repulsion term in Eq. (1) and leads to a completely new parametrization. We consider that to be beyond the scope of this study. Regarding the difference in results at the peak

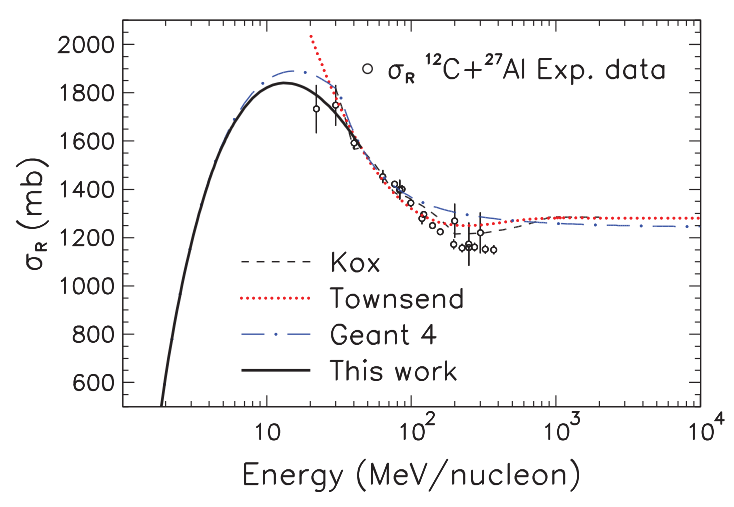

FIG. 3. (Color online) The energy dependence of total reaction cross sections, predicted with the different versions of the Kox and Shen models, for ${ }^{12} \mathrm{C}+{ }^{27} \mathrm{Al}$. Experimental data, shown as open circles, are taken from Ref. [21].

value around a few tens of $\mathrm{MeV} /$ nucleon, our approach looks smoother and more physically correct considering the general energy dependence of nucleus-nucleus total reaction cross sections in that energy region.

Projectile-target asymmetry. We also point out that the Kox and Shen models have a projectile-target asymmetry, i.e.,

$$
\sigma_{R}(\mathrm{p}+\mathrm{t}) \neq \sigma_{R}(\mathrm{t}+\mathrm{p}) \quad \text { for } \quad A_{i} \neq 2 Z_{i}(i=\mathrm{p}, \mathrm{t}),
$$

where $\mathrm{p}$ and $\mathrm{t}$ denotes the projectile and target, respectively. This is due to the last term in Eq. (4), which was introduced by Kox et al. as an option for heavy target nuclei. Shen et al. made it permanent in their model, but it is not obvious from Ref. [6] that this anomaly exists, and it may therefore cause unexpected results for the user. For systems with both a heavy projectile and a heavy target the difference is small, but for systems where either the projectile or target is a proton or ${ }^{3} \mathrm{He}$ the difference is significant. Figure 5 shows an example for protons on ${ }^{28} \mathrm{Si}$. The lines show the result of the Shen model with our modified parametrization: the solid black line for the case that the proton is the projectile and the dashed (red) line for ${ }^{28} \mathrm{Si}$ as the projectile. As can be seen, the model works much better for the case with the proton as projectile. In some cases, such as for protons on ${ }^{12} \mathrm{C}$, both options give

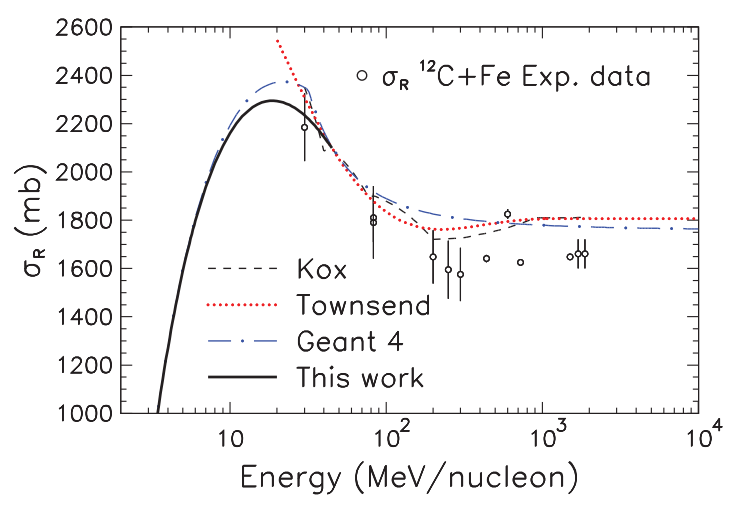

FIG. 4. (Color online) The energy dependence of total reaction cross sections, predicted with the different versions of the Kox and Shen models, for ${ }^{12} \mathrm{C}+\mathrm{Fe}$. Experimental data, shown as open circles, are taken from Ref. [21]. 


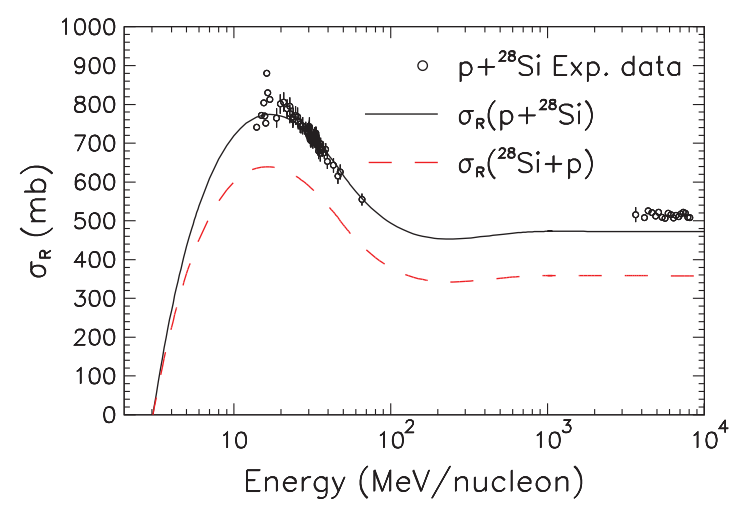

FIG. 5. (Color online) Total reaction cross sections for the system $p+{ }^{28} \mathrm{Si}$ predicted with the Shen model using the modified parametrization of this work. The black line shows the proton as projectile while the dashed (red) line shows the case when the proton is treated as the target. Experimental data are taken from Refs. [22,23].

somewhat ambiguous results. But based on clear cases such as the one in Fig. 5, our advice is that the lighter particle always should be treated as the projectile, when using the Kox or Shen models. Such a function is already implemented in the PHITS code [24].
Conclusions. We have proposed a modified parametrization of the transparency parameter in the Kox and Shen models of total reaction cross sections. Our modification is based on the parametrizations used by Townsend and Wilson [17] and by the GEANT4 [18-20] collaboration, and improves the usage of the Kox [4] and Shen [6] model by giving a smoother energy dependence of the cross sections. Furthermore it extends the models to be used for projectile energies below $30 \mathrm{MeV} /$ nucleon. We also give the recommendation that due to an inherent projectile-target asymmetry the models should be used so that the lighter nucleus is always treated as the projectile, no matter what the actual case might be.

More experimental data are needed to benchmark and improve the existing total reaction cross-section models. From the available experimental data it is not possible to determine which of the models displayed in the present work give the most accurate prediction of total reaction cross sections over the entire energy range.

Acknowledgments. Part of this work is supported by the RIKEN iTHES project. L.S. greatly acknowledges A.K. for his kind support that made it possible for Sihver to stay two months at RIKEN, Japan. M.L. work is partly funded by a grant from JSPS.
[1] C. Perrin, S. Kox, N. Longequeue, J. B. Viano, M. Buenerd, R. Cherkaoui, A. J. Cole, A. Gamp, J. Menet, R. Ost, R. Bertholet, C. Guet, and J. Pinston, Phys. Rev. Lett. 49, 1905 (1982).

[2] S. Kox, A. Gamp, R. Cherkaoui, A. J. Cole, N. Longequeue, J. Menet, C. Perrin, and J. B. Viano, Nucl. Phys. A 420, 162 (1984).

[3] S. Kox, A. Gamp, C. Perrin, J. Arvieux, R. Bertholet, J. F. Bruandet, M. Buenerd, Y. El Masri, N. Longequeue, and F. Merchez, Phys. Lett. B 159, 15 (1985).

[4] S. Kox, A. Gamp, C. Perrin, J. Arvieux, R. Bertholet, J. F. Bruandet, M. Buenerd, R. Cherkaoui, A. J. Cole, Y. El-Masri, N. Longequeue, J. Menet, F. Merchez, and J. B. Viano, Phys. Rev. C 35, 1678 (1987).

[5] H. L. Bradt and B. Peters, Phys. Rev. 77, 54 (1950).

[6] W. Q. Shen, B. Wang, J. Feng, W. L. Zhan, Y. T. Zhu, and E. P. Feng, Nucl. Phys. A 491, 130 (1989).

[7] R. K. Tripathi, F. A. Cucinotta, and J. W. Wilson, Nucl. Instrum. Methods B 117, 347 (1996).

[8] R. K. Tripathi, J. W. Wilson, and F. A. Cucinotta, Nucl. Instrum. Methods B 129, 11 (1997).

[9] R. K. Tripathi, F. A. Cucinotta, and J. W. Wilson, Nucl. Instrum. Methods B 155, 349 (1999).

[10] V. Andersen, F. Ballarini, G. Battistoni, M. Campanella, M. Carboni, F. Cerutti, A. Empl, A. Fassò, A. Ferrari, E. Gadioli, et al., Adv. Space Res. 34, 1302 (2004).

[11] G. Battistoni, S. Muraro, P. R. Sala, F. Cerutti, A. Ferrari, S. Roesler, A. Fassò and J. Ranft, AIP Conf. Proc. 896, 31 (2007).

[12] A. Ferrari, P. R. Sala, A. Fassò, and J. Ranft, CERN Report No. CERN-2005-10; INFN Report No. INFN/TC_05/11; SLAC Report No. SLAC-R-773, 2005.
[13] L. Sihver, C. H. Tsao, R. Silberberg, T. Kanai, and A. F. Barghouty, Phys. Rev. C 47, 1225 (1993).

[14] L. Sihver and D. Mancusi, Radiat. Meas. 44, 38 (2009).

[15] M. Takechi, M. Fukuda, M. Mihara, K. Tanaka, T. Chinda, T. Matsumasa, M. Nishimoto, R. Matsumiya, Y. Nakashima, H. Matsubara et al., Phys. Rev. C 79, 061601(R) (2009)

[16] K. Iida, A. Kohama, and K. Oyamatsu, J. Phys. Soc. Jpn. 76, 044201 (2007).

[17] L. W. Townsend and J. W. Wilson, Phys. Rev. C 37, 892 (1988).

[18] S. Agostinelli, J. Allison, K. Amako, J. Apostolakis, H. Araujo, P. Arce, M. Asai, D. Axen, S. Banerjee, G. Barrand et al., Nucl. Instrum. Methods A 506, 250 (2003).

[19] J. Allison, K. Amako, J. Apostolakis, H. Araujo, P. Arce Dubois, M. Asai, G. Barrand, R. Capra, S. Chauvie, R. Chytracek et al., IEEE Trans. Nucl. Sci. 53, 270 (2006).

[20] GEANT4 Physical Reference Manual [http://geant4.web.cern.ch/ geant4/support/userdocuments.shtml].

[21] L. Sihver, M. Lantz, T. T. Böhlen, A. Mairani, A. F. Cerutti, and A. Ferrari, in Proc. 2012 IEEE Aerospace Conf. (IEEEAC) (IEEE, Piscataway, NJ, 2012).

[22] R. F. Carlson, Atomic Data Nucl. Data Tab. 63, 93 (1996).

[23] B. M. Bobchenko, A. E. Buklei, A. V. Vlasov, I. I. Vorobev, L. S. Vorobev, N. A. Goryainov, Yu. G. Grishuk, O. B. Gushchin, B. L. Druzhinin, V. V. Zhurkin et al., Yad. Fiz. 30, 1553 (1979) [Sov. J. Nucl. Phys. 30, 805 (1979)].

[24] T. Sato, K. Niita, N. Matsuda, S. Hashimoto, Y. Iwamoto, S. Noda, T. Ogawa, H. Iwase, H. Nakashima, T. Fukahori, K. Okumura, T. Kai, S. Chiba, T. Furuta, and L. Sihver, J. Nucl. Sci. Technol. 50, 913 (2013). 